\title{
Armed Kyphoplasty: An Indirect Central Canal Decompression Technique in Burst Fractures
}

\author{
(D) A. Venier, (D) Roccatagliata, (D) M. Isalberti, (D). Scarone, (DD.E. Kuhlen, (D) M. Reinert, (D) G. Bonaldi, (D).A. Hirsch, and
} (D. Aianfoni

\begin{abstract}
BACKGROUND AND PURPOSE: Burst fractures are characterized by middle column disruption and may feature posterior wall retropulsion. Indications for treatment remain controversial. Recently introduced vertebral augmentation techniques using intravertebral distraction devices, such as vertebral body stents and Spinejack, could be effective in fracture reduction and fixation and might obtain central canal clearance through ligamentotaxis. This study assesses the results of armed kyphoplasty using vertebral body stents or SpineJack in traumatic, osteoporotic, and neoplastic burst fractures with respect to vertebral body height restoration and correction of posterior wall retropulsion.
\end{abstract}

MATERIALS AND METHODS: This was a retrospective assessment of 53 burst fractures with posterior wall retropulsion and no neurologic deficit in 51 consecutive patients treated with armed kyphoplasty. Posterior wall retropulsion and vertebral body height were measured on pre- and postprocedural CT. Clinical and radiologic follow-up charts were reviewed.

RESULTS: Armed kyphoplasty was performed as a stand-alone treatment in 43 patients, combined with posterior instrumentation in 8 and laminectomy in 4. Pre-armed kyphoplasty and post-armed kyphoplasty mean posterior wall retropulsion was 5.8 and 4.5 $\mathrm{mm}$, respectively $(P<.001)$, and mean vertebral body height was 10.8 and $16.7 \mathrm{~mm}$, respectively $(P<.001)$. No significant clinical complications occurred. Clinical and radiologic follow-up (1-36 months; mean, 8 months) was available in 39 patients. Three treated levels showed a new fracture during follow-up without neurologic deterioration, and no retreatment was deemed necessary.

CONCLUSIONS: In the treatment of burst fractures with posterior wall retropulsion and no neurologic deficit, armed kyphoplasty yields fracture reduction, internal fixation, and indirect central canal decompression. In selected cases, it might represent a suitable minimally invasive treatment option, stand-alone or in combination with posterior stabilization.

ABBREVIATIONS: AKP = armed kyphoplasty; BKP = balloon kyphoplasty; PWR = posterior wall retropulsion; SAIF = Stent-Screw Assisted Internal Fixation; VAS = Visual Analog Scale; VBH = vertebral body height; VBS = vertebral body stenting; SJ = SpineJack

T horacolumbar burst fractures can result from axial-load high-energy trauma or even from minor trauma if bone is weakened by osteoporosis or neoplasm. Burst fractures are characterized by a high degree of osseous fragmentation, outward fragment dispersion, and middle column disruption and may be

Received April 9, 2019; accepted after revision August 28.

From the Departments of Neurosurgery (A.V., P.S., D.E.K., M.R.) and Neuroradiology (L.R., M.I., A.C.), Neurocenter of Southern Switzerland, Lugano, Switzerland; Department of Neuroradiology (G.B.), Papa Giovanni XXIII Hospital, Bergamo, Italy; Department of Neurosurgery (G.B.), Clinica Igea, Milan, Italy; Department of Neuroradiology (J.A.H.), Massachusetts General Hospital, Harvard Medical School, Boston, Massachusetts; and Department of Neuroradiology (A.C.), Inselspital, University Hospital of Bern, Bern, Switzerland.

Please address correspondence to Alice Venier, MD, Neurocenter of Southern Switzerland, Via Tesserete, 46, 6900 Lugano, Switzerland; e-mail:

alice.venier@eoc.ch

http://dx.doi.org/10.3174/ajnr.A6285 associated with posterior wall retropulsion (PWR) in the central canal. Burst fractures are considered unstable, carrying a risk for immediate or delayed neurologic compromise. ${ }^{1}$

In practice, treatment of burst fractures, especially without neurologic injury, remains controversial, with indications ranging from conservative ${ }^{2}$ to complex combined ventral and dorsal surgical approaches. ${ }^{3}$ Conservative treatment may imply long periods of diminution of the activities of daily living. Moreover, burst fractures carry the risk of progressive focal kyphosis and neurologic deterioration. ${ }^{4}$ Conversely, surgical treatment should stabilize the vertebral body, restoring vertebral body height (VBH) and alignment, correcting kyphosis, and decompressing the central canal, ${ }^{5,6}$ thereby reducing pain and allowing early mobilization.

To address these goals, traditional pedicle-screw instrumentation allows indirect fracture and kyphosis reduction, ${ }^{7,8}$ and via a dorsal approach, the central canal can be decompressed by 
laminectomy and posterior wall fragment impaction ${ }^{9,10}$ or indirectly restored through a posterior external cantilever and distraction maneuver, exploiting ligamentotaxis of the posterior longitudinal ligament. ${ }^{11}$ Nevertheless, stabilization of the anterior column remains crucial in these fractures to avoid loss of correction and instrumentation failure. ${ }^{12}$ Surgical anterior instrumentation with strut grafting, mesh cage, and plates has proved effective in stabilizing the anterior column ${ }^{13,14}$ but requires a more invasive approach, which could be associated with increased morbidity. ${ }^{15}$

A minimally invasive approach would be desirable and might represent a balanced compromise. Cement augmentation, mainly with a balloon kyphoplasty (BKP) technique, as a stand-alone or in combination with posterior instrumentation, has been proposed as an option, ${ }^{16-19}$ but it might be not very effective in correcting kyphosis, due to the potential loss of height restoration at balloon deflation. ${ }^{20,21}$ Moreover, in the presence of PWR, BKP might be unable to clear the canal and is even considered relatively contraindicated due to the risk of epidural cement leakage and further displacement of bony fragments in the central canal, potentially leading to worsening of the neurologic condition. ${ }^{22,23}$ More recently introduced percutaneous intrasomatic distraction devices, such as SpineJack (SJ; Stryker, Kalamazoo, Michigan) and vertebral body stents (VBSs; DePuy-Synthes, Johnson \& Johnson, Raynham, Massachusetts) used to perform an armed kyphoplasty (AKP) might be able to overcome the deflation effect of BKP and allow a minimally invasive stabilization of the vertebral body. ${ }^{24,25}$ An effective internal vertebral body fracture reduction and fixation might, in turn, allow a ligamentotaxis effect and canal clearance.

In this study, we retrospectively assessed the results of AKP using VBS or SJ, with or without posterior instrumentation, in traumatic, osteoporotic, and neoplastic burst fractures with regard to correction of $\mathrm{PWR}$ and restoration of $\mathrm{VBH}$.

\section{MATERIALS AND METHODS Patient Population}

All the patients who underwent AKP at Neurocenter of Southern Switzerland between August 2013 and December 2017 were considered for the study. Inclusion criteria were the following: 1) the presence of traumatic, osteoporotic (spontaneous or related to minor trauma), or neoplastic burst fracture without neurologic deficits, 2) the presence of a retropulsed bone fragment in the central canal documented on the preprocedural CT, and 3) a postprocedural CT scan obtained within 10 days of treatment. The local ethics committee approved this study.

\section{Procedure}

AKP was performed with the patient under general anesthesia using VBS (Figs 1-3) or SJ (Figs 3 and 4) under biplane fluoroscopic guidance. The procedure was conducted using standard techniques for either device. ${ }^{24,25}$ VBS AKP was performed standalone or with the additional insertion of pedicular screws anchoring the stents in accordance with the recently reported StentScrew Assisted Internal Fixation (SAIF) technique (Figs 2 and $3){ }^{26}$ Intraoperative myelography was used in selected cases of

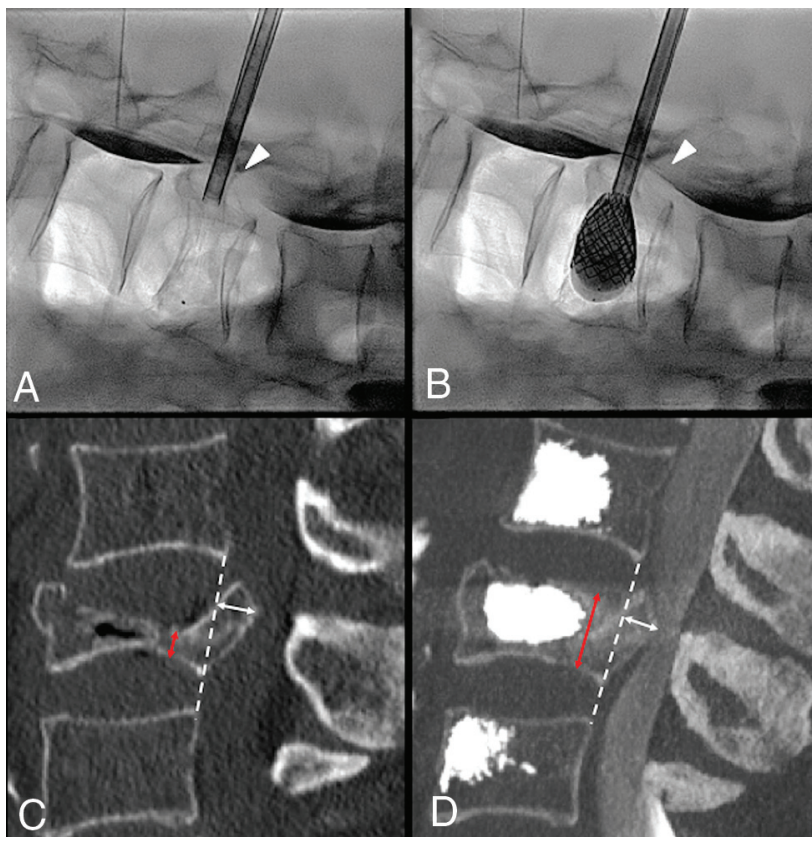

FIG 1. Severe L3 vertebral body collapse $(A)$ in a 74-year-old woman with osteopenia following minor trauma. Intraoperative myelogram through intradural injection of contrast agent at L1-L2 (arrow, A) shows an opacification defect of the dural sac dorsal to the retropusled fragment at L3 (arrowhead, A). On fracture reduction through balloon-expanded vertebral body stents $(B)$, the myelogram shows greater opacification of the dural sac at L3 (arrowhead, B), a real-time indirect sign of ligamentotaxis and partial central canal clearance. $C$ and $D$, Preoperative and postoperative midsagittal $C T$ images used for measurement of vertebral body height at the maximum point of collapse (red arrows) and of posterior wall retropulsion (white arrows) perpendicular to the dashed white line connecting the postero-inferior corner of the cranial vertebral body and the posterosuperior corner of the caudal one, representing the expected original posterior wall, now intersecting the PWR.

lumbar fractures to monitor central canal stenosis during the procedure. When deemed necessary, AKP was performed in combination with surgical posterior stabilization, either with percutaneous or open surgery with or without decompressive laminectomy (Fig 4), but without additional distraction or posterior wall fragment impaction. When deemed appropriate by the operator in patients with osteoporosis, prophylactic vertebral augmentation was performed at the adjacent levels. ${ }^{27}$ The individual treatment decision and approach were chosen via a multidisciplinary spine board.

\section{Measurements}

PWR and VBH were measured on pre- and postprocedural CT scans by 2 readers, a neuroradiologist and a neurosurgeon, in consensus. Images were reformatted with orthogonal multiplanar reconstructions, with a section thickness of $2 \mathrm{~mm}$, interval of $2 \mathrm{~mm}$, and bone algorithm, on a PACS system. A straight line was drawn on the midsagittal plane from the posterior-inferior corner of the cranial to the posterior-superior corner of the caudal adjacent vertebral bodies, ideally representing the original position of the normal prefracture posterior wall of the target level. This line intersected the retropulsed fractured posterior 


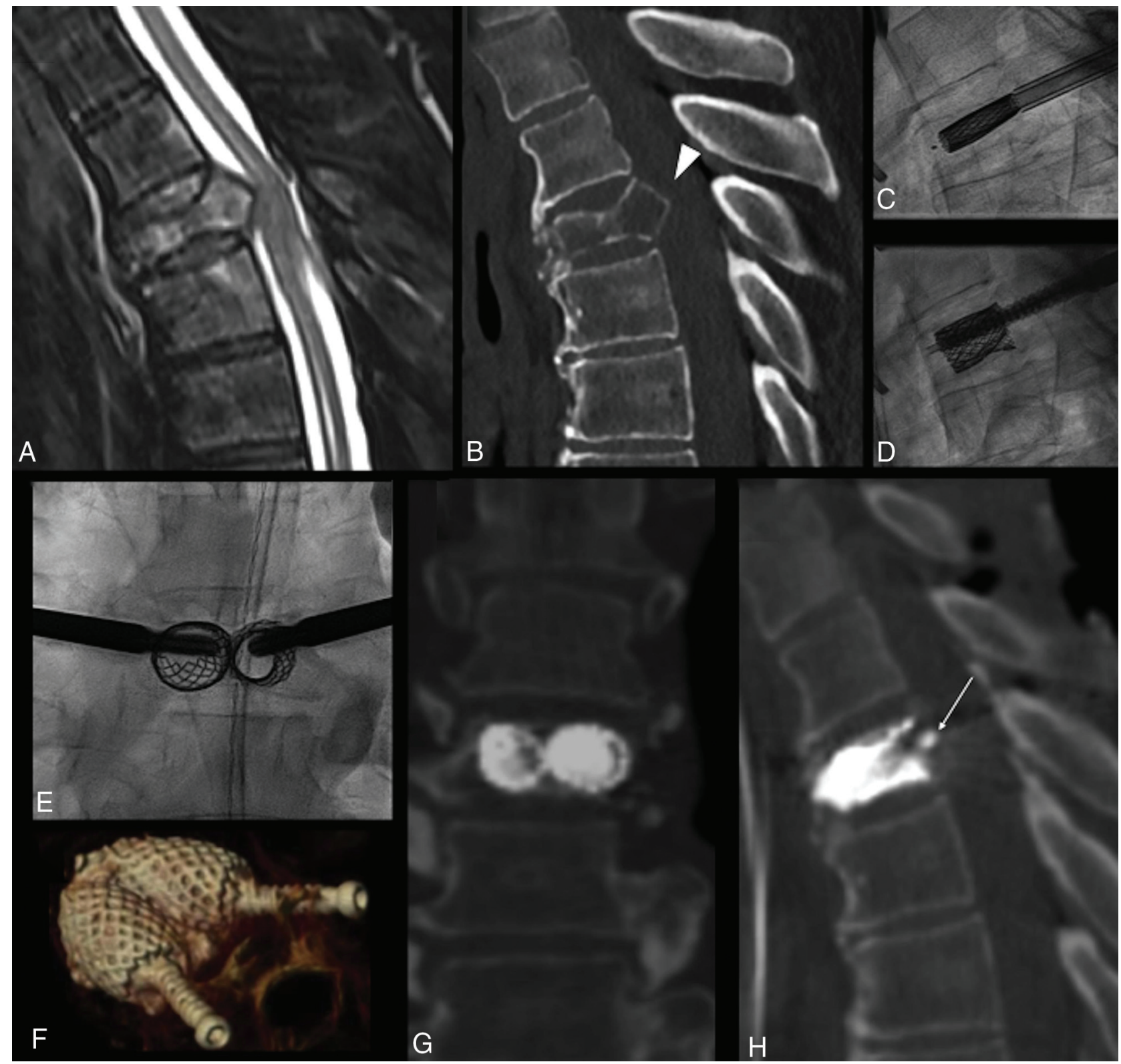

FIG 2. Lung cancer metastatic T4 fracture in a 67-year-old man, with disabling back pain. MR imaging $(A)$ and $C T(B)$ show a lytic lesion, with vertebral body collapse and retropulsion of an osseous fragment (arrowhead, B), causing spinal cord compression, but the patient was neurologically intact. The patient underwent armed kyphoplasty with the SAIF technique $(C-E)$ with bilateral stent and screw implant, with a decompressive surgery backup plan on standby. The procedure was uneventful, and the patient showed no neurologic worsening. Postoperative CT $(F-H)$ shows a 3D view of the stent-screw-cement complex $(F)$ and, most notably, the vertebral body height restoration and correction of posterior wall retropulsion (arrow, $H$ ) through ligamentotaxis. The patient was ambulating the same day and could undergo radiation treatment during the following days.

wall. The PWR was then measured perpendicularly from this posterior wall line on the midsagittal image (Fig 1). VBH measurement was obtained on the midsagittal image from the superior-to-inferior endplates at the most collapsed point (Fig 1).

\section{Statistical Analysis}

Statistical analysis was performed using SPSS 20.0 (IBM, Armonk, New York).

For non-normally distributed variables, we used the relatedsamples Wilcoxon signed rank test to compare the median preoperative-versus-postoperative degree of PWR and to compare median preoperative-versus-postoperative VBH. Visual Analog Scale (VAS) scores for pain intensity at baseline, at 1 month, and at 6 months' follow-up were also compared using the same nonparametric test.

\section{Follow-Up}

Every patient underwent plain film and CT of the spine within 10 days from treatment to evaluate the procedure results. Clinical assessment after the procedure was mainly directed to assess for 
neurologic deterioration. In a subgroup of patients, extended imaging and clinical follow-up were available and were reviewed to evaluate long-term target-level stability results, new vertebral fractures, neurologic status stability, and other clinical conditions requiring a new treatment. The VAS pain score (0-10) assessment preprocedure and at 1 and 6 months postprocedure was available for a subgroup of patients.

\section{RESULTS}

\section{Patient Population}

Of 193 patients, 94 patients were excluded because the fracture was not associated with a retropulsed bone fragment; 48 were excluded because either a pre- or a postprocedure CT scan was not available for analysis. The patient population, fulfilling all inclusion criteria, therefore included 51 patients (female/male ratio: 34:17; age range, $46-90$ years; mean, 73 years) with thoracic $(20 / 53)$ or lumbar $(33 / 53)$ fractures. The most frequently treated levels were T12 and L1 (23/53). Two patients were treated at 2 levels. The fractures were traumatic in $32 / 53$, osteoporotic in $12 / 53$, and neoplastic in 9/53 cases.

\section{Procedure}

AKP was performed with VBS at 46/53 levels and with SJ at 7/53 levels. VBS AKP was performed with the SAIF technique at 33/46 levels. Intraoperative lumbar myelography was performed in 4 cases (Fig 1).

Concurrent posterior surgical stabilization with pedicular screws and rods was performed in $8 / 51$ patients along with decompressive laminectomy in $4 / 8$.

In 1 case, an epidural cement leak occurred causing L4 radicular pain, which promptly resolved after steroid nerve block. One patient experienced transient and completely reversible paraparesis, without evidence of worsening central canal compromise and without epidural cement leaks on postprocedure CT and MR imaging. No further intraprocedural clinical complications occurred. No other patient showed worsening neurologic status after the procedure or at follow-up.

\section{PWR and VBH}

There was a statistically significant difference between the degree of PWR preoperatively (mean, $5.8 \pm 2 \mathrm{~mm}$; range, $2-10 \mathrm{~mm}$ ) and postoperatively (mean, $4.5 \pm 1.9 \mathrm{~mm}$; range, $0-9.4 \mathrm{~mm}$; $P<.001$ ), and there was a statistically significant difference between the $\mathrm{VBH}$ preoperatively (mean, $10.7 \pm 4.4 \mathrm{~mm}$; range, $2-21 \mathrm{~mm}$ ) and postoperatively (mean, $16.5 \pm 3.8 \mathrm{~mm}$; range, $7.7-23.6 \mathrm{~mm} ; P<.001$ ). When we compared pre- and postoperative CT scans, the PWR difference ranged between +2 and $-4 \mathrm{~mm}$ (mean, $-1.2 \mathrm{~mm}$ ) and the mean gain of $\mathrm{VBH}$ was $5.8 \mathrm{~mm}$.

Individual case analysis showed that 41/53 levels had PWR correction, 6/53 had unchanged PWR, and 6/53 had worsened PWR postoperatively, while 51/53 had some degree of VBH restoration and 2/53 showed reduced $\mathrm{VBH}$ at postprocedure CT.

\section{Follow-Up}

Beyond the postprocedure clinical assessment, spine plain films, and CT within 10 days, 39/51 patients (41/53 levels) had an extended clinical and imaging follow-up, at least with standing spine plain films, at multiple and variable time points, ranging from 1 to 36 months postprocedure (mean, 8 months). In 19/41 (46\%) levels, the postprocedure VBH was fully maintained; in 19/ 41 (46\%), mild subsidence of the superior or inferior endplates was noted (Fig 3) with no convincing impact on alignment and kyphosis, while in 3/41 (8\%), a recurrent VBH collapse of the target level was noted. In the follow-up group, 22/41 levels were studied with a cross-sectional imaging technique (8 with MR imaging and CT, 11 with CT, and 3 with MR imaging), and PWR could be assessed. Fourteen of 22 showed stability of the PWR correction compared with the postoperative CT, while $8 / 22$ showed a recurrence in PWR. Of these 8 cases, 2 were associated with refracture of the target level, while 6 were associated with subsidence of the treated vertebra at follow-up. No retreatment was necessary at AKP-treated target levels. Clinical follow-up showed no neurologic deterioration.

Preprocedural and follow-up VAS pain scores were available for $31 / 51$ patients. The mean VAS score at baseline was $8.5 \pm 1.1$ (range, 6-10); at 1-month follow-up, it was $4.0 \pm 2.1$ (range 0-9); and at 6 months' follow-up, it was $2.8 \pm 1.8$ (range, $0-7$ ). In this cohort, the VAS scores at baseline versus 1 month and versus 6 months were significantly different $(P<.001)$.

\section{DISCUSSION}

In this study, AKP using recently introduced vertebral body fracture internal distraction devices such as VBS and SJ was safely able to obtain VBH restoration and PWR correction in traumatic (Figs 3 and 4), osteoporotic (Figs 1 and 3), and neoplastic burst fractures (Fig 2). It was used as a stand-alone minimally invasive procedure in most cases or in combination with a posterior surgical approach (Fig 4), but without the need to perform any direct form of PWR correction. This minimally invasive approach had only 2 periprocedural complications, both with benign clinical resolution; showed durable results at follow-up; and required no re-intervention on the target level.

There is no definite consensus on the management of burst fractures with PWR. Some authors support a conservative approach in neurologically intact patients, claiming the possible spontaneous remodeling and resorption of the posterior wall osseous fragment encroaching the central canal, ${ }^{28}$ while others suggest a variety of surgical approaches, including decompressive laminectomy, stabilization of the anterior column combined with a dorsal instrumentation, ${ }^{13,15}$ and direct or indirect repositioning of retropulsed bone fragments. ${ }^{9,10}$

The goals of treatment are to obtain early patient mobilization and a painless, balanced, stable vertebral column with maximum spine mobility and optimal neurologic function. In neurologically intact patients, the different surgical techniques are not necessarily superior to a nonoperative approach. ${ }^{6}$ These results might be influenced by the potentially significant morbidity and increased cost of an anterior column reconstructive surgery and by the failure rate of stand-alone posterior surgical fracture reduction and stabilization. ${ }^{11,12} \mathrm{~A}$ safe, effective, and durable minimally invasive solution to reduce and stabilize the fracture might perform differently and better approach the ideal treatment goals. 


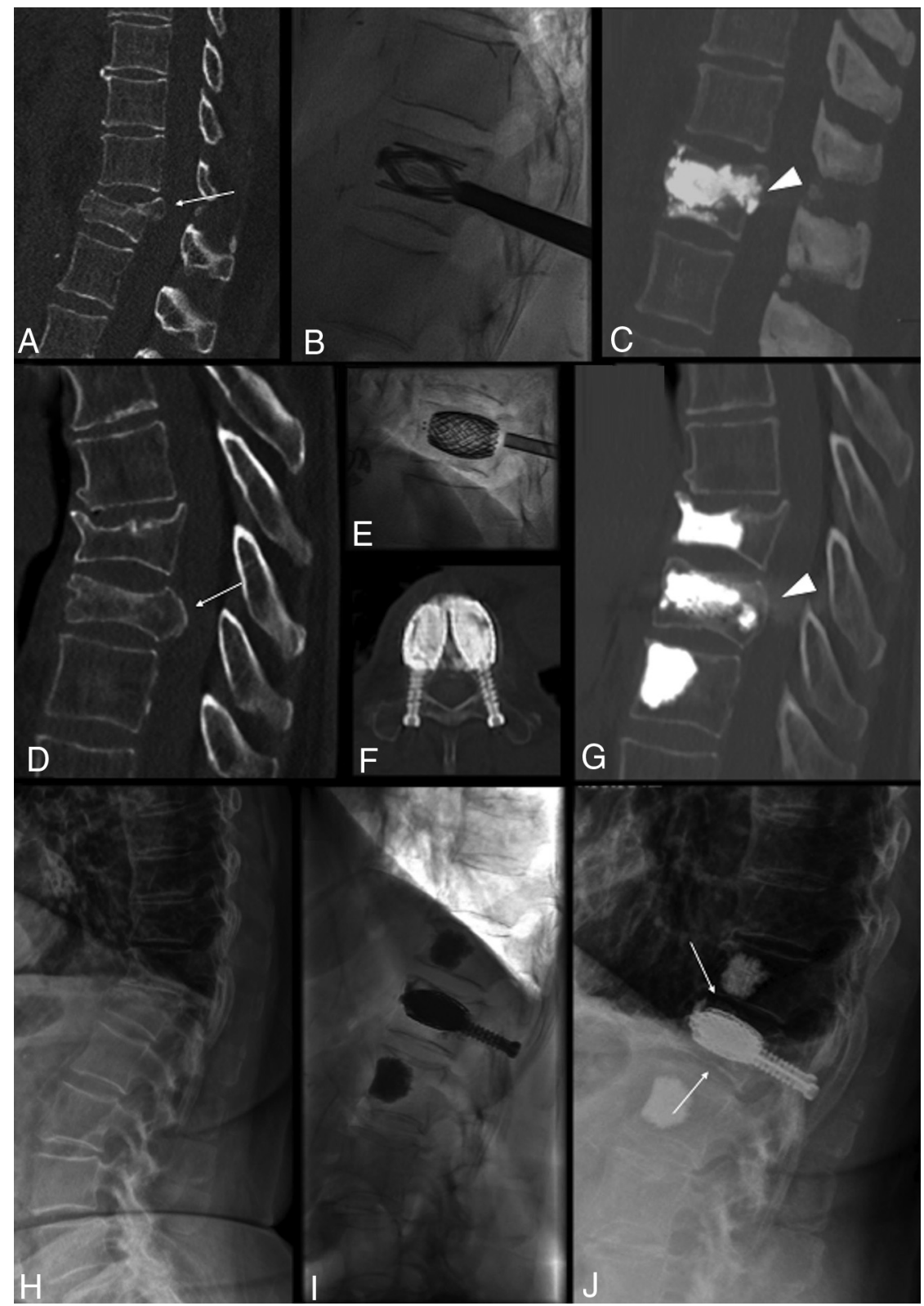

FIG 3. Three different cases $(A-C),(D-G)$, and $(H-J)$. A-C, Treatment with the Spinejack of a traumatic incomplete burst fracture of $\mathrm{T} 12$ in a 55-year-old man with posterior wall retropulsion (arrow, A) and junctional kyphosis. Postoperative CT shows vertebral height restoration, central canal clearance through retropulsed fragment correction (arrowhead, C), and kyphosis correction. D-G, Treatment with the SAIF technique of a traumatic T10 fracture in a 78year-old man with osteoporosis with $>50 \%$ height loss and posterior wall retropulsion (arrow, D), with effective height restoration and posterior wall reposition (arrowhead, G). $\mathrm{H}-J$, Treatment of an L1 osteoporotic fracture with bone subsidence (arrows, J) around the cement cast at 1-month follow-up, not compromising alignment and curvature. The patient was asymptomatic.

BKP has been used to treat burst fractures, especially in a combined approach with dorsal instrumentation, ${ }^{17,18}$ but its potential to effectively obtain $\mathrm{VBH}$ restoration has been questioned $^{20,21}$ and might even be relatively contraindicated. ${ }^{29}$ With all that in mind, in clinical practice, BKP is likely used relatively frequently, given the extreme pain and functional limitations of patients for extended times. VBS and SJ have been reported as an alternative to BKP to reduce the deflation effect and potentially guarantee more reliable height restoration in wedge-shaped or incomplete burst compression fractures. ${ }^{30-32} \mathrm{~A}$ recent randomized controlled trial showed better kyphosis correction, maintained at 12 months for SJ versus BKP in osteoporotic compression fractures. ${ }^{33} \mathrm{~A}$ cadaveric study ${ }^{34}$ has shown the ability of SJ to reposition the retropulsed posterior wall of a burst fracture model and substantially maintain this gain after cyclic recompression, while posterior instrumentation alone did not maintain central canal clearance, but the potential of AKP to restore $\mathrm{VBH}$ and correct the PWR in burst fractures has not been investigated in vivo. In fact, most studies reporting the use of VBS and SJ have focused on wedge compression fractures of an osteoporotic nature, ${ }^{25,30,31}$ and fewer have dealt with incomplete burst fractures. ${ }^{32}$ Within these studies, pain outcome was typically the primary end point, while kyphosis or VBH correction was secondary. In general, repositioning of the posterior fragments is underrepresented in most evaluations. ${ }^{34}$

AKP can effect VBH restoration, avoiding height loss due to deflation effect, and is increasingly used as a standalone measure to reconstruct and restore axial-load capability in traumatic, osteoporotic, and malignant fractures. ${ }^{26,35}$ As a consequence of the internal fracture distraction and kyphosis reduction, AKP appears to allow ligamentotaxis correction of the PWR without the need to perform external distraction through a posterior instrumentation or even more invasive maneuvers of fragment repositioning through direct impaction. In this study, we included 8 neoplastic fractures that had a retropulsed bone fragment (Fig 2), while we did not include cases with epidural nonosseous soft-tissue masses. An epidural soft-tissue mass might, in fact, behave differently from an osseous PWR and would have been more difficult to measure on postoperative CT. Intraoperative myelography, already described in the setting of vertebral augmentation procedures at risk for central canal encroachment, ${ }^{36}$ was used in only a minority of cases in this series, but it seemed potentially useful in selected patients to have a visual control under fluoroscopy of the PWR and to directly demonstrate the effect of ligamentotaxis during fracture reduction (Fig 1). 


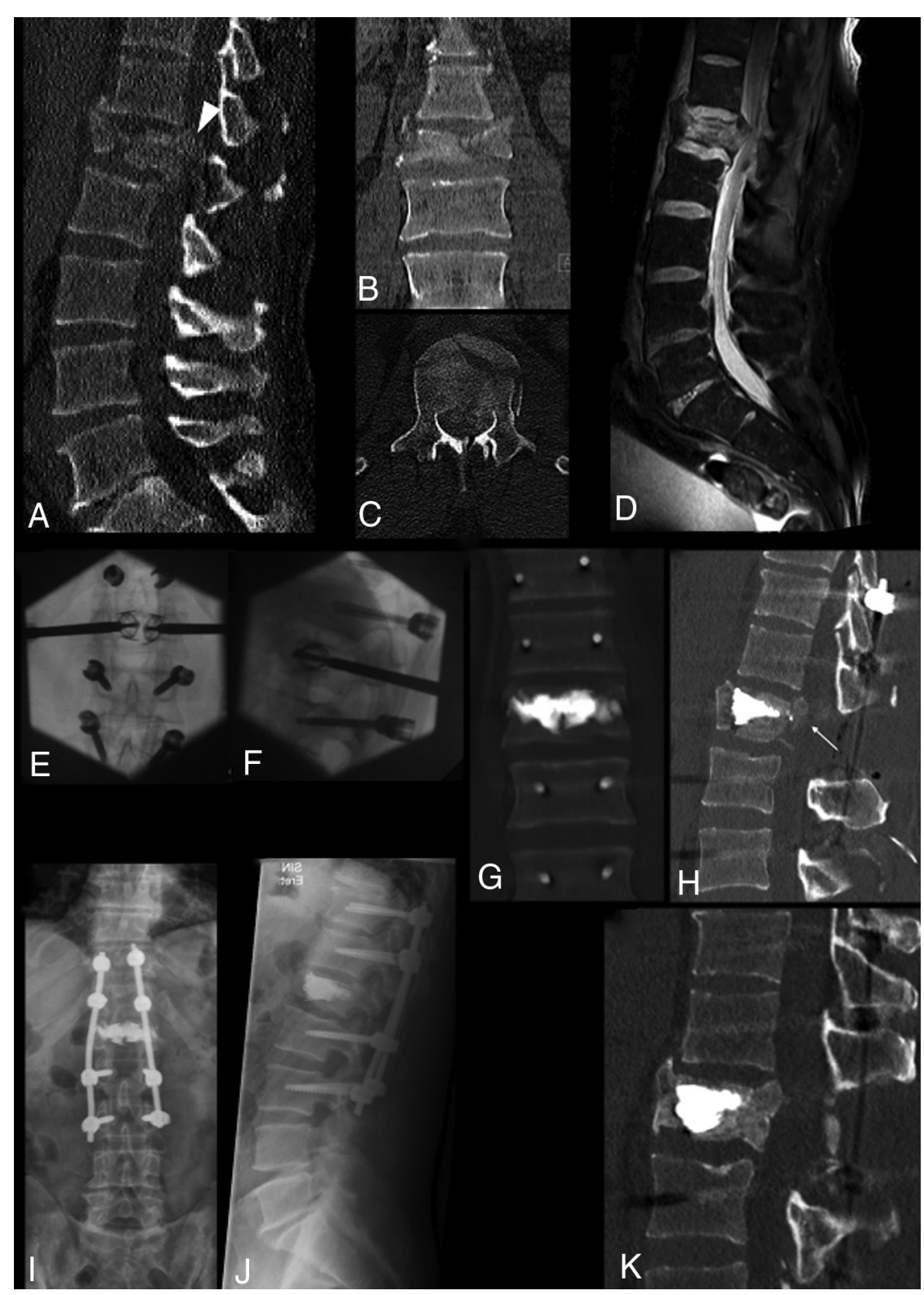

FIG 4. Complete burst fracture of L1 following high-energy trauma in a 40-year-old man with marked vertebral body fragmentation $(A-C)$ and posterior wall retropulsion (arrowhead, $A$ ). Sagittal fat-saturated proton density MR image $(D)$ shows an epidural hematoma and compression of the conus medullaris, but the patient was neurologically intact. The patient underwent surgical treatment in a hybrid operation room, including, in sequence, L1 decompressive laminectomy, pedicular screw placement, fracture reduction and vertebral body augmentation with percutaneous bilateral Spinejack, and posterior stabilization with spinal rods $(E$ and $F)$. Postprocedural CT ( $G$ and $H$ ) shows fracture and kyphosis reduction and, most important, central canal clearance (arrow, $H$ ) through ligamentotaxis. Notably, no maneuvers of direct fragment impaction or of posterior fracture distraction were performed. Follow-up imaging with standing plain films at 6 months ( $I$ and $/$ ) and with CT at 12 months $(K)$ shows preserved vertebral body height and alignment and osseous healing around the cement cast. In this case, a more invasive procedure of corpectomy and anterior column stabilization could be successfully avoided by armed kyphoplasty.

We found a statistically significant difference between the mean degree of PWR and VBH pre- and postoperatively, which suggests the biomechanical effectiveness of the technique.

Besides the statistically significant postoperative changes of PWR and VBH, we found 2 cases in which VBH was reduced postoperatively, 6 cases with worsened PWR, and 6 cases with unchanged PWR. Explanations for such technical failures are not clear. Worsening PWR can certainly be an undesired effect of the internal vertebral body distraction, as generally feared, if ligamentotaxis does not occur; but fracture, $\mathrm{VBH}$, and PWR might have also negatively evolved in the time lapse between preoperative imaging and the procedure. Unchanged PWR might also be related to osseous healing and/or nonefficient ligamentotaxis, which might not have allowed fragment repositioning. In addition, the group of patients we analyzed was heterogeneous, having included traumatic, osteoporotic, and malignant fractures, which theoretically may respond differently to AKP. While it is difficult to relate the technical efficacy in $\mathrm{VBH}$ restoration and PWR correction observed in this cohort to a definite measure of clinical benefit, PWR is still considered a relative contraindication to vertebral augmentation. Not infrequently, it represents an argument for open stabilization surgery. The results of this study might serve to mitigate the fear that AKP might worsen the status of neurologically intact patients with burst fractures. In the group of patients with available VAS assessment, there was significant and sustained pain reduction as expected compared with previously published larger series using similar treatment techniques. $^{36}$ In the 41 levels with available follow-up, the results obtained with AKP were confirmed to be stable at a mean follow-up of 8 months (range, 1-36 months) because in 38/ 41, the postoperative $\mathrm{VBH}$ was either stable or showed only minimal endplate subsidence (Fig 3); only in 3 cases did we encounter a refracture of the vertebral body treated with AKP, with VBH loss. As to a PWR correction, 14/22 patients who had cross-sectional imaging follow-up available showed stable PWR at follow-up. Some degree of PWR recurrence was noted in $8 / 30$, associated with recurrent collapse $(2 / 8)$ or subsidence. Patients with refracture of the target level and/or PWR recurrence presented with mild or no new symptoms at clinical follow-up, did not show neurologic deterioration, and did not require any further invasive treatment. 
One might consider subsidence of the endplates and minimal PWR recurrence as nearly physiologic changes after AKP, namely because the surrounding fractured and weakened bone of the vertebral body on weight-bearing loading remodels and might undergo resorption phenomena against the new rigid internal scaffold, represented by VBS and SJ with polymethylmethacrylate, but usually these outcomes do not have clinical significance. The 3 new collapses reported in this series occurred in elderly patients treated for traumatic (1/3) and osteoporotic (2/3) fractures, all in the context of nontreated osteopenia. The importance of a thorough management of frequently underlying osteopenia or osteoporosis in patients at risk remains critical to reduce new fracture risk or hardware failure. ${ }^{37}$

AKP was performed using VBS in 46 levels, and SJ, in the remaining 7. We tend to use SJ for AKP when bone mass is preserved, especially in young patients with traumatic mechanism of fracture and impacted morphology of the fracture, to the impacted morphology needs a powerful internal fracture distraction, while we rather use VBS in bone of poor quality with a high degree of vertebral body fragmentation, osteoporosis, and lytic lesions, in which the vertebral body rather needs an internal scaffold to restore its stability and axial-load capability. ${ }^{38}$

There are several limitations to the present study, including its retrospective design, the small size and heterogeneity of the sample, and nonsystematic follow-up. There might have been a selection bias in the studied patient series, but the decision to treat with AKP versus a standard surgical approach was reached for every individual patient by a multidisciplinary spine board. The inclusion in this series of patients treated in combination with a posterior surgical approach underscores the possibility of treating even severe burst fractures with AKP, avoiding surgical stabilization of the anterior column and more invasive maneuvers to clear the central canal.

Given the small number of patients and confounding factors, including concurrent surgical interventions, fracture etiology heterogeneity, and technical differences in performing the AKP procedure, the conclusions of our analysis need to be confirmed in larger prospective studies.

\section{CONCLUSIONS}

AKP appears to represent a viable technique to treat neurointact burst fractures with PWR, in combination with posterior instrumentation or in selected cases as a stand-alone procedure, being able to effect $\mathrm{VBH}$ restoration and indirect central canal decompression through PWR correction. This minimally invasive approach should offer durable results and thus represents an alternative to avoid more invasive anterior column stabilization interventions and retropulsed bone fragment reposition techniques.

Disclosures: Pietro Scarone-UNRELATED: Consultancy: Depuy Synthes J\&J Medical Devices.* Joshua A. Hirsch—UNRELATED: Board Membership: Ceranovus (Data and Safety Monitoring Board service), Relievant (Data Monitoring Committee service); Consultancy: Medtronic, Comments: ongoing consulting. *Money paid to the institution.

\section{REFERENCES}

1. Denis F. Spinal instability as defined by the three-column spine concept in acute spinal trauma. Clin Orthop Relat Res 1984:65-76 Medline
2. Mumford J, Weinstein JN, Spratt KF, et al. Thoracolumbar burst fractures: the clinical efficacy and outcome of nonoperative management. Spine (Phila Pa 1976) 1993;18:955-70 CrossRef

3. Payer M. Unstable burst fractures of the thoraco-lumbar junction: treatment by posterior bisegmental correction/fixation and staged anterior corpectomy and titanium cage implantation. Acta Neurochir (Wien) 2006;148:299-306; discussion 306 CrossRef Medline

4. Rajasekaran S. Thoracolumbar burst fractures without neurological deficit: the role for conservative treatment. Eur Spine J 2010;19:4047 CrossRef Medline

5. Khurjekar $\mathrm{Hk} \mathrm{K}$, Kardile $\mathrm{M}$. Treatment algorithm for unstable burst fracture. International Journal of Spine 2016;1:27-32

6. Scheer JK, Bakhsheshian J, Fakurnejad S, et al. Evidence-based medicine of traumatic thoracolumbar burst fractures: a systematic review of operative management across 20 years. Global Spine J 2015;5:73-82 CrossRef Medline

7. Aebi M, Etter C, Kehl T, et al. Stabilization of the lower thoracic and lumbar spine with the internal spinal skeletal fixation system: indications, techniques, and first results of treatment. Spine (Phila $\mathrm{Pa}$ 1976) 1987;12:544-51 CrossRef

8. Dick W, Kluger P, Magerl F, et al. A new device for internal fixation of thoracolumbar and lumbar spine fractures: the "fixateur interne." Paraplegia 1985;23:225-32 CrossRef Medline

9. Oro J, Watts C, Gaines R. Vertebral body impactor for posterior lateral decompression of thoracic and lumbar fractures: technical note. J Neurosurg 1989;70:285-86 CrossRef Medline

10. Park SH, Kim SD, Moon BJ, et al. Short segment percutaneous pedicle screw fixation after direct spinal canal decompression in thoracolumbar burst fractures: an alternative option. J Clin Neurosci 2018;53:48-54 CrossRef Medline

11. Whang PG, Vaccaro AR. Thoracolumbar fracture: posterior instrumentation using distraction and ligamentotaxis reduction. J Am Acad Orthop Surg 2007;15:695-701 CrossRef Medline

12. Lakshmanan P, Jones A. Mehta Recurrence of kyphosis and its functional implications after surgical stabilization of dorsolumbar unstable burst fractures. Spine J 2009;9:1003-09 CrossRef Medline

13. Machino M, Yukawa Y, Ito K, et al. Posterior/anterior combined surgery for thoracolumbar burst fractures-posterior instrumentation with pedicle screws and laminar hooks, anterior decompression and strut grafting. Spinal Cord 2011;49:573-79 CrossRef Medline

14. Zhang S, Thakur JD, Khan IS, et al. Anterior stabilization for unstable traumatic thoracolumbar spine burst fractures. Clin Neurol Neurosurg 2015;130:86-90 CrossRef Medline

15. Oprel PP, Tuinebreijer WE, Patka P, et al. Combined anterior-posterior surgery versus posterior surgery for thoracolumbar burst fractures: a systematic review of the literature. Open Orthop J 2010;4:93-100 CrossRef Medline

16. Kruger A, Zettl R, Ziring E, et al. Kyphoplasty for the treatment of incomplete osteoporotic burst fractures. Eur Spine J 2010;19:893900 CrossRef Medline

17. Korovessis P, Hadjipavlou A, Repantis T. Minimal invasive short posterior instrumentation plus balloon kyphoplasty with calcium phosphate for burst and severe compression lumbar fractures. Spine (Phila Pa 1976) 2008;33:658-67 CrossRef Medline

18. Korovessis P, Repantis T, Petsinis G, et al. Direct reduction of thoracolumbar burst fractures by means of balloon kyphoplasty with calcium phosphate and stabilization with pedicle-screw instrumentation and fusion. Spine (Phila $\mathrm{Pa}$ 1976) 2008;33:E100-08 CrossRef Medline

19. Verlaan JJ, Somers I, Dhert WJ, et al. Clinical and radiological results 6 years after treatment of traumatic thoracolumbar burst fractures with pedicle screw instrumentation and balloon assisted endplate reduction. Spine J 2015;15:1172-78 CrossRef Medline

20. Lee JH, Lee DO, Lee JH, et al. Comparison of radiological and clinical results of balloon kyphoplasty according to anterior height loss 
in the osteoporotic vertebral fracture. Spine J 2014;14:2281-89 CrossRef Medline

21. Disch AC, Schmoelz W. Cement augmentation in a thoracolumbar fracture model: reduction and stability after balloon kyphoplasty versus vertebral body stenting. Spine (Phila $\mathrm{Pa}$ 1976) 2014;39: E1147-53 CrossRef Medline

22. Walter J, Haciyakupoglu E, Waschke A, et al. Cement leakage as a possible complication of balloon kyphoplasty-is there a difference between osteoporotic compression fractures (AO type A1) and incomplete burst fractures (AO type A3.1)? Acta Neurochir 2012;154:313-19 CrossRef Medline

23. Saliou G, Rutgers DR, Kocheida EM, et al. Balloon-related complications and technical failures in kyphoplasty for vertebral fractures. AJNR Am J Neuroradiol 2010;31:175-79 CrossRef Medline

24. Rotter R, Martin H, Fuerderer S, et al. Vertebral body stenting: a new method for vertebral augmentation versus kyphoplasty. Eur Spine J 2010;19:916-23 CrossRef Medline

25. Renaud C. Treatment of vertebral compression fractures with the cranio-caudal expandable implant SpineJack(R): technical note and outcomes in 77 consecutive patients. Orthop Traumatol Surg Res 2015;101:857-59 CrossRef Medline

26. Cianfoni A, Distefano D, Isalberti M, et al. Stent-screw-assisted internal fixation: the SAIF technique to augment severe osteoporotic and neoplastic vertebral body fractures. I Neurointerv Surg 2018;11:603-09 CrossRef Medline

27. Diel P, Freiburghaus L, Roder C, et al. Safety, effectiveness and predictors for early reoperation in therapeutic and prophylactic vertebroplasty: short-term results of a prospective case series of patients with osteoporotic vertebral fractures. Eur Spine $J$ 2012;21:792-99 CrossRef Medline

28. de Klerk LW, Fontijne WP, Stijnen T, et al. Spontaneous remodeling of the spinal canal after conservative management of thoracolumbar burst fractures. Spine (Phila Pa 1976) 1998;23:1057-60 CrossRef Medline

29. Chandra RV, Meyers PM, Hirsch JA, et al. Vertebral augmentation: report of the Standards and Guidelines Committee of the Society of NeuroInterventional Surgery. J Neurointerv Surg 2014;6:7-15 CrossRef Medline
30. Noriega DC, Rodríguez-Monsalve F, Ramajo R, et al. Long-term safety and clinical performance of kyphoplasty and SpineJack(R) procedures in the treatment of osteoporotic vertebral compression fractures: a pilot, monocentric, investigator-initiated study. Osteoporos Int 2019;30:637-45 CrossRef Medline

31. Werner CM, Osterhoff G, Schlickeiser J, et al. Vertebral body stenting versus kyphoplasty for the treatment of osteoporotic vertebral compression fractures: a randomized trial. J Bone Joint Surg Am 2013;95:577-84 CrossRef Medline

32. Hartmann F, Griese M, Dietz SO, et al. Two-year results of vertebral body stenting for the treatment of traumatic incomplete burst fractures. Minim Invasive Ther Allied Technol 2015;24:161-66 CrossRef Medline

33. Noriega D, Maestretti G, Renaud C, et al. Clinical performance and safety of 108 SpineJack implantations: 1-year results of a prospective multicentre single-arm registry study. Biomed Res Int 2015;2015:1. CrossRef Medline

34. Kruger A, Oberkircher L, Figiel J, et al. Height restoration of osteoporotic vertebral compression fractures using different intravertebral reduction devices: a cadaveric study. Spine J 2015;15:1092-98 CrossRef Medline

35. Cianfoni A, Distefano D, Pravata E, et al. Vertebral body stent augmentation to reconstruct the anterior column in neoplastic extreme osteolysis. J Neurointerv Surg 2019;11:313-18 CrossRef Medline

36. Cianfoni A, Raz E, Mauri S, et al. Vertebral augmentation for neoplastic lesions with posterior wall erosion and epidural mass. AJNR Am J Neuroradiol 2015;36:210-18 CrossRef Medline

37. Bae JS, Park JH, Kim KJ, et al. Analysis of risk factors for secondary new vertebral compression fracture following percutaneous vertebroplasty in patients with osteoporosis. World Neurosurg 2017;99:387-94 CrossRef Medline

38. Cianfoni A, Distefano D, Pravata E, et al. Vertebral body stent augmentation to reconstruct the anterior column in neoplastic extreme osteolysis. J Neurointer Surg 2019;11:313-18 CrossRef Medline 